\title{
Ultrafast spin crossover in a single crystal
}

\author{
Ryan L. Field ${ }^{1,2}$, Lai Chung Liu ${ }^{1,2}$, Yifeng Jiang ${ }^{1,2}$, Wojciech Gawelda ${ }^{3}$, Cheng Lu ${ }^{1}$, and $R$. \\ J. Dwayne Miller ${ }^{1, *}$, \\ ${ }^{1}$ Departments of Chemistry and Physics, University of Toronto, 80 St. George Street, Toronto, \\ Ontario, M5S 3H6, Canada \\ ${ }^{2}$ Center for Free Electron Laser Science, Max Planck Institute for the Structure and Dynamics of \\ Matter, Bld. 99, Luruper Chaussee 149, 22761 Hamburg, Germany \\ ${ }^{3}$ European XFEL GmbH, Holzkoppel 4, 22869 Schenefeld, Germany
}

\begin{abstract}
Femtosecond spectroscopy and electron diffraction are used to characterize spin crossover in single crystal iron(II)-tris(bipyridine)bis(hexafluorophosphate). The high-spin lifetime is reduced compared to in solution. Preliminary electron diffraction experiments show evidence of ultrafast $\mathrm{Fe}-\mathrm{N}$ bond elongation associated with spin crossover and the subsequent molecular reorganization resulting from vibrational cooling.
\end{abstract}

\section{Introduction}

Spin crossover ( $\mathrm{SCO}$ ) is a process whereby the spin state of a molecular system transitions between low-spin (LS) and high-spin (HS) states by changes in pressure/temperature or by photoexcitation. Iron(II)-tris(bipyridine) $\left(\left[\mathrm{Fe}^{\mathrm{II}}(\mathrm{bpy})_{3}\right]^{2+}\right)$ is an $\mathrm{SCO}$ complex has been extensively studied in the solvated phase using transient absorption (TA) in the UV and visible ranges [1] and time-resolved X-ray absorption [2] and emission [3] spectroscopies. It has generally been concluded that, upon absorption of a photon, the LS complex is excited to a metal-to-ligand charge transfer (MLCT) band from which it undergoes several intersystem crossing and/or internal conversion steps before arriving in the HS state on a sub-picosecond time-scale. The HS complex then undergoes vibrational cooling over a few picoseconds, before ultimately relaxing back to the LS state with a lifetime of $\sim 650 \mathrm{ps}$. Fast oscillatory signals have been observed in reported data, which were attributed to coherent vibrational motions on the HS surface. The majority of studies on the $\left[\mathrm{Fe}^{\mathrm{II}}(\mathrm{bpy})_{3}\right]^{2+}$ complex have used solvated samples. Herein, we report on the dynamics in single crystal Iron(II)-tris(bipyridine)-bis(hexafluorophosphate) ([Fe $\left.\left(\mathrm{Fpy}_{3}\right]\left(\mathrm{PF}_{6}\right)_{2}\right)$ using femtosecond transient absorption (TA) spectroscopy [4]. Femtosecond electron diffraction (FED) studies require single crystal samples because of the greater spatial resolution that they provide. We also report on preliminary FED studies currently in progress which will separate nuclear dynamics from electronic effects. The results of our TA studies show that the SCO transition dynamics in the single crystal environment are unchanged on the ultrafast time scale compared to the those in the solvated case, but that the lifetime of the HS is significantly reduced due to chemical pressure exerted by the lattice.

*Corresponding author: dwayne.miller@mpsd.mpg.de 


\section{Experimental Methods}

The TA spectrometer [4] has an instrument response function of $80 \mathrm{fs}$. Thin slices of $\left[\mathrm{Fe}^{\mathrm{II}}(\mathrm{bpy})_{3}\right]\left(\mathrm{PF}_{6}\right)_{2}(100-400 \mathrm{~nm})$ were microtomed from bulk crystal samples and mounted on $0.5 \mathrm{~mm}$ thick sapphire substrates for measurement. The polarizations of the $400 \mathrm{~nm}$ pump and supercontinuum probe were parallel and set to maximize the absorption of the pump light. The fluence of the pump at the sample position was approximately $3 \mathrm{~mJ} / \mathrm{cm}^{2}$. All measurements were carried out at room temperature. Similar conditions were used for fs electron diffraction studies, albeit with a lower excitation fluence of approximately 1.3 $\mathrm{mJ} / \mathrm{cm}^{2}$, using the protocols described previously [5].

\section{Results and Discussion}

The TA data in the UV and visible ranges over short and long time-scales are shown in figure 1. The short-lived MLCT state is apparent as excited state absorption (ESA) in the visible range at wavelengths $<460 \mathrm{~nm}$ and $>580 \mathrm{~nm}$ immediately after time-zero. Persistent ground state bleach (GSB) in the visible and ESA in the UV are observed, which signify the depletion of the LS state and population of the HS state, respectively [1]. The HS ESA band blueshifts over a few picoseconds. Fast oscillations are observed in both the UV and visible ranges. On the long time-scale, the dynamics are more complex than in the solvated case and cannot be described by a single exponential decay. Oscillations with periods of hundreds of picoseconds are also observed on this time-scale.

To analyze this data, we employed a global analysis approach which resulted in fits consisting of the sum of a small number of exponentially decaying spectral components (decay-associated spectra, DAS) convolved with the instrument response function [4]. The DAS are shown in figure 2. DAS with decay constants on the order of $100 \mathrm{fs}$ were found, which can be associated with the decay of the MLCT state. The UV range showed a $1.7 \mathrm{ps}$ DAS which can be interpreted as resulting from vibrational cooling or structural rearrangement of the molecule following the spin transition [1,2,5]. The components which are effectively infinite on this time-scale reflect the population of the HS state and corresponding depletion of the LS state. On the longer time-scale, we see components which decay with a $\sim 100$ ps time constant. The spectrum of the component in the visible range is very similar to the inverted ground state absorption spectrum and these can thus be straightforwardly associated with the decay of the HS state back to the ground state. The lifetime is significantly shorter than the $\sim 650$ ps lifetime observed in the solvated case due to chemical pressure exerted by the lattice, which destabilises the HS state. The effectively infinite component in the visible range strongly resembles the second derivative of the ground state absorption spectrum, which suggests a line-broadening mechanism. We interpret the effectively infinite components on this time-scale as broadening of the spectra due to heat deposited in the lattice during photoexcitation.

The Fourier transforms (FT) of the global fit's residuals for the short-time scans are shown in figure 3 . These reveal complex oscillatory behaviour on this time-scale in both the UV and visible ranges, with a greater number of significant frequencies than in the solvated case. Frequencies are seen at 55, 81, 139 and $165 \mathrm{~cm}^{-1}$ which correspond to a combination of vibrations on the HS state surface and impulsively stimulated Raman oscillations in the ground state. The periods of the slow oscillations are the result of acoustic phonons in the crystal lattice, with significant changes in the spectral peaks illustrating the very strong electron-phonon coupling in this system.

Preliminary results of femtosecond electron diffraction studies are shown in figure 4. Preliminary analysis of associated time traces shows changes in the molecular structure on 
two time scales. To identify the origin of the two steps observed, the temporally-resolved changes in the diffraction pattern were compared to changes simulated using the known LS state structure and a simulated HS structure based on previous simulations of the same in liquid [5]. By isolating the contributions of two key dynamical motions, the nature of the two steps observed can be deduced. We observe a subpicosecond instrument responselimited step that our simulations suggest results from the ultrafast $\mathrm{Fe}-\mathrm{N}$ bond length expansion that is typical in many photoinduced SCO complexes. A second step, which occurs over several picoseconds, can similarly be attributed to ligand motions leading to molecular reorganization via vibrational cooling. These results are generally in good agreement with previously reported results on a number of SCO compounds $[1,2,4]$. However, they are in contrast to FED results on a related compound [Fe(PM$\left.\mathrm{AzA})_{2}\right](\mathrm{NCS})_{2}$, which showed no subpicosecond step [6]. More detailed analysis of these results is underway and a full manuscript is in preparation.

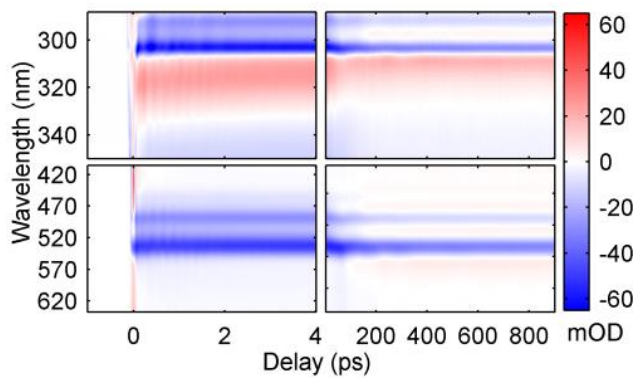

Fig. 1. TA of $\left[\mathrm{Fe}^{\mathrm{II}}(\mathrm{bpy})_{3}\right]\left(\mathrm{PF}_{6}\right)_{2}$ in the $\mathrm{UV}$ and visible.

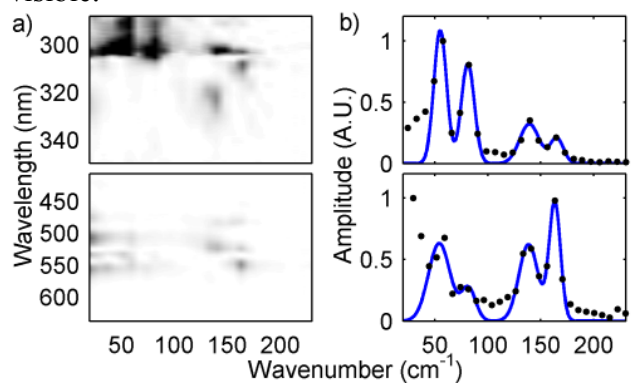

Fig. 3. FT of residuals of global fit. a) Spectrally resolved FT and b) spectrally integrated FT in $\mathrm{UV}$ and visible.

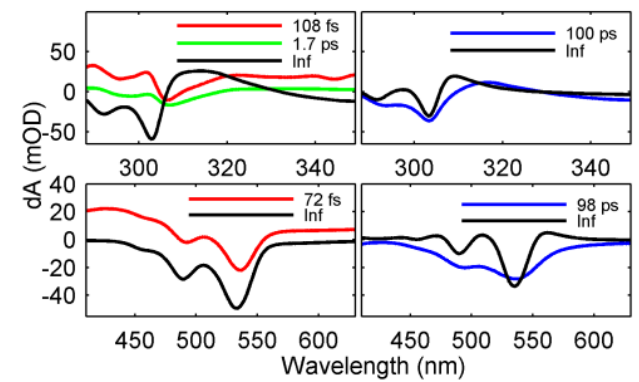

Fig. 2. DAS of global fit of TA data in the UV and visible on short and long time scales.

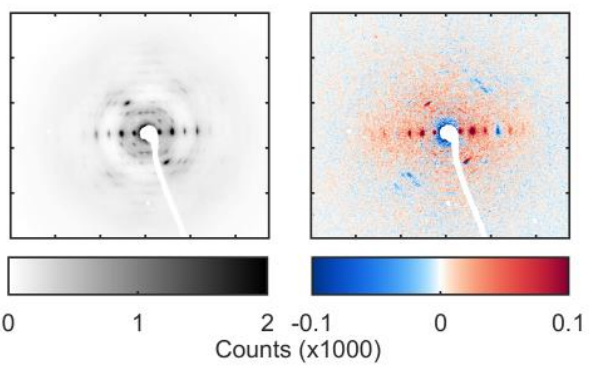

Fig. 4. Static diffraction pattern of $\left[\mathrm{Fe}^{\mathrm{II}}(\mathrm{bpy})_{3}\right]\left(\mathrm{PF}_{6}\right)_{2}$ (left) and difference diffraction pattern averaged from 10-50 ps (right).

The TA results presented here show that the ultrafast SCO in single crystal is a local molecular process very similar to that observed in solution, with differences caused by the crystal environment only apparent on the time scale of hundreds of picoseconds. The preliminary FED data represent the first direct measurement of the structural SCO dynamics of $\left[\mathrm{Fe}^{\mathrm{II}}(\mathrm{bpy})_{3}\right]\left(\mathrm{PF}_{6}\right)_{2}$ with atomic resolution over the entire molecule.

\section{References}

1. G. Auböck and M. Chergui, Nat. Chem., 7, 629 (2015).

2. H. T. Lemke et al., Nat. Comm., 8, 15342 (2017).

3. W. Zhang et al., Nature, 509, 345 (2014).

4. R.L. Field et al., Chem. Eur. J., 22, 5118 (2016)

5. L. M. Lawson Daku,and A. Hauser, J. Phys. Chem. Lett. 1, 1830-1835 (2010).

6. Y. Jiang et al., Angew. Chem. Int. Ed., 56, 7130 (2017). 